Strychnin - Jodmethylnitrat. Dieses Nitrat wird gebildet durch Wechselwirkung von Strychnin-Jodmethyljodid mit Silbernitrat in alkoholischer oder in heiss gesättigter wässeriger Lösung und darauf folgendes freiwilliges Verdunstenlassen der erzielten Lösungen. Es resultieren hierbei gut ausgebildete, farblose Nadeln, welche bei $100^{\circ} \mathrm{C}$. nichts an Gewicht verloren. Obschon dieses Nitrat wiederholt dargestellt wurde und auch ausserlich durchaus einheitlich aussah, wurde doch der Jodgehalt nach Carius stets etwas zn niedrig gegen die $\mathrm{Be}-$ rechnung gefunden:

J 22,$0 ; 22,27 ; 21,52 ; 20,60 ; 21,83 \%$.

Berechnet für $\mathrm{C}^{21} \mathrm{H}^{22} \mathrm{~N}^{2} \mathrm{O}^{2} \cdot \mathrm{CH}^{2} \mathrm{~J} \cdot \mathrm{NO}^{2}: 23,56 \stackrel{\alpha}{o} \mathrm{~J}$.

\title{
126. Ueber das Verhalten des Jodoforms und Chloroforms gegen Strychnin.
}

Von P. F. Trowbridge.

I. Jodoform.

Eine Verbindung des Strychnins mit Jodoform : $3 \mathrm{C}^{21} \mathrm{H}^{22} \mathrm{~N}^{2} \mathrm{O}^{2} \cdot \mathrm{CHJ}^{3}$, ist bereits von Lextrait') dargestellt. Dieser Autor löste zur Gewinnung dieses Produktes Strychnin und Jodoform unter Erwărmen in Alkohol auf und stellte diese Lösung zur Krystallisation beiseite, wobei direkt die Abscheidung in Krystallen erfolgte.

Lextrait giebt an, dass die von ihm erhaltene Jodoformverbindung nicht beständig sei, indem schon bei $90^{\circ} \mathrm{C}$., ebenso beim Behandeln derselben mit Alkohol Zersetzung stattfindet. In Aether und Chloroform soll diese Jodoformverbindung leicht löslich sein, jedoch soll sich in diesen Lösungen bald freies Jod ausscheiden.

Ich habe die Einwirknng des Jodoforms auf Strychnin, im Anschluss an Versuche, die ich auf Veranlassung von Herrn Geh. Rat E. Schmidt uber das Verhalten des Methylenjodids zu Strychnin ausftihrte ${ }^{2}$ ), von neuem studiert, namentlich um die Zersetzungsprodukte dieser Jodoformverbindungen kennen zu lernen.

Zur Darstellung des Jodoform-Strychnins löste ich die Komponenten in Chloroform auf und brachte dann diese, gleiche Molekülmengen enthaltenden Lösungen zusammen. Hierbei erfolgte weder direkt, noch

1) Compt. rend. 92, 1057.

2) S. vorstehende Abhandlung. 
nach 24 stündigem Stehen eine Abscheidung von Krystallen. Erst nach Zusatz von Aether entstand ein rotbrauner, krystallinischer Niederschlag. Letzterer wurde gesammelt, durch Absaugen und Auswaschen mit Aether von der Mutterlauge befreit und dann bei gewöhnlicher Temperatur getrocknet. Die Analyse dieser Vérbindung lehrte, dass in letzterer ebenfalls nur ein Körper der Formel $3 \mathrm{C}^{21} \mathrm{H}^{22} \mathrm{~N}^{2} \mathrm{O}^{2}$. $\mathrm{CHJ}^{8}$ vorlag, obschon die Komponenten zu gleichen Molekülen angewendet worden waren.

Zur Analyse dieses Produktes wurden $0,3867 \mathrm{~g}$ desselben in einem

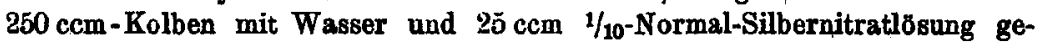
schüttelt und das Gemisch nach einiger Zeit dann auf dem Wasserbade erwärmt. Nach den Erkalten wurde die Mischung zur Marke mit Wasser aufgefüllt, nach dem Absetzen filtriert und in $100 \mathrm{ccm}$ des Filtrats der Ueberschuss an Silbernitrat durch $1 / 10$-Normal-Rhodanlösung zurücktitriert. Es wurden hierbei $6,6 \mathrm{ccm}$ verbraucht.
Gefunden:
J 27,80
Berechnet für $3 \mathrm{C}^{21} \mathrm{H}^{22} \mathrm{~N}^{2} \mathrm{O}^{2} \cdot \mathrm{CHJ}^{3}$ : 27,29 .

Obschon die Zusammenset\%ung und die äusseren Eigenschaften dieser Verbindung mit den Angaben von Lextrait ubereinstimmten, machte sich doch insofern ein beinerkenswerter Unterschied kenntlich, als der von mir erLaltene Körper sehr beständig zu sein schien. Nachdem die Verbindung 24 Stunden, nur mit einem Uhrglase bedeckt, an der Luft gelegen hatte und darauf 5 Stunden noch bei $100^{\circ} \mathrm{C}$. erbitzt worden war, enthielt dieselbe noch immer 27,31\% Jod.

In Alkobol löste sich obige Verbindung nur schwierig auf und zwar fand dabei gleichzeitig eine Zersetzung derselben statt.

Als eine Probe obiger Verbindung nur gelinde mit Alkohol erwärmt und das Gelöste von dem Ungelösten alsdann abfiltriert wurde, resultierten beim Erkalten des Filtrats rotbraune Blättchen, welche jedoch nur noch 26,01 \% Jod enthielten. Bei einem zweiten Versuche wurde sogar nur eine Verbindung mit einem Jodgehalte von 24,23\% erhalten.

Wesentlich anders gestaltete sich das Resultat, als die Verbindung $3 \mathrm{C}^{21} \mathrm{H}^{22} \mathrm{~N}^{2} \mathrm{O}^{2} \cdot \mathrm{CHJ}^{3}$ mit Alkohol gekocht wurde. Hierbei schieden sich aus dem Filtrat, nachdem dasselbe bis auf ein Drittel eingedampft war, dunkelrotbraune Blättchen aus, welche $36,13 \%$ Jod enthielten. Der von dem kochenden Alkohol nicht gelöste Teil wurde getrocknet, in Chloroform gelost und diese Lossung mit Aether versetzt. Hierdurch erhielt ich weiter ein dunkel rotbrannes, krystallinisches Pulver, welches sich bei $100^{\circ} \mathrm{C}$. nicht veränderte. Der Jodgehalt dieser Verbindung war der gleiche, wie derjenige der aus Alkohol ausgeschiedenen Blättchen: $35,95 \%$. 


\section{2. \\ J $\quad 36,13 \quad 35,95$ \\ Berechnet für $2 \mathrm{C}^{21} \mathrm{H}^{22} \mathrm{~N}^{2} \mathrm{O}^{2} \cdot \mathrm{CHJ}^{3}$ :}

Aus diesen Daten geht hervor, dass die Verbindung $3 \mathrm{C}^{21} \mathrm{H}^{22} \mathrm{~N}^{2} \mathrm{O}^{2}$. $\mathrm{CH}^{3}$ beim Kochen mit Alkohol eine Zersetzung erleidet unter Bildung des viel stabileren Produktes $2 \mathrm{C}^{21} \mathrm{H}^{22} \mathrm{~N}^{2} \mathrm{O}^{2}$. CH. ${ }^{8}$.

Da beide Jodoformverbindungen des Strychnins beim Digerieren mit wässeriger Silbernitratlösung den gesamten Gehalt an Jod als Jodsilber abgeben, so dürfte hieraus hervorgehen, dass in diesen Additionsprodukten das Jod noch als $\mathrm{CH}^{8}$ vorhanden ist.

Im Kapillarrohr erhitzt, zeigten beide Verbindungen bei $150^{\circ} \mathrm{C}$. äusserlich noch keine Veränderung; bei $200^{\circ} \mathrm{C}$. trat Schwärzung und bei etwa $220^{\circ}$ C. Schmelzen ein.

\section{Chloroform.}

Beim Zusammenbringen von Strychnin and Chloroform zeigte sich, dass beide Körper sich bei gewöhnlicher Temperatur nicht verbinden, weder wenn das Alkaloid direkt in Chloroform gelöst, noch wenn eine wässerige Strychninsalzlösung, nach Zusatz von Kalihydrat, mit Chloroform ausgeschüttelt wird. Auch durch Erhitzen einer Auflösung von Strychnin in Chloroform auf $100^{\circ}$ konnte eine Verbindung beider Körper nicht erzielt werden. Anders gestalteten sich jedoch die Verhältnisse, als die Temperatur noch gesteigert wurde.

Eine Chloroform-Strychninlösung, $1: 10$ bereitet, schied nach dreistündigem Erhitzen im geschlossenen Rohre auf $120^{\circ} \mathrm{C}$. nach dem Erkalten bereits einige prismatische Krystalle aus. Die Menge der letzteren nahm sehr bedeutend $\mathrm{zu}$, als die Temperatur auf $150^{\circ} \mathrm{C}$. gesteigert und das Erhitzen 10 Stunden lang fortgesetzt wurde. Die ausgeschiedenen Krystalle sahen nach dem Auswaschen mit Chloroform fast farblos aus, während die von denselben abgegossene Chloroformlösung braun gefärbt war.

Die auf letztere Weise erhaltenen Krystalle lösten sich in Chloroform, Alkohol und Wasser auf. Die Beständigkeit derselben war nur eine geringe. Nachdem dieselben so lange an der Luft getrocknet waren, bis ein Geruch nach Chloroform nicht mehr zu bemerken war, liess sich nach dreistündiger Aufbewahrung in einem geschlossenen Gefässe ein solcher sehr kräftig wieder konstatieren. Die Analyse der lufttrockenen Verbindung ergab folgende Werte:

1. $0,3318 \mathrm{~g}$ Substanz lieferten nach Carius $0,3532 \mathrm{~g} \mathrm{Ag} \mathrm{Cl}=26,32 \% \mathrm{Cl}$.

2. $0,3673 \mathrm{~g}$ Substanz, in Wasser gelöst und mit Silbernitrat gefällt, lieferten $0,1084 \mathrm{~g} \mathrm{Ag} \mathrm{Cl}=7,29 \% \mathrm{Cl}$. $7,29 \% \mathrm{Cl}$.

3. $0,4388 \mathrm{~g}$ Substanz ergaben in wässeriger Lösung $0,1294 \mathrm{~g} \mathrm{Ag} \mathrm{Cl}=$ 
Für eine Verbindung $\mathrm{C}^{21} \mathrm{H}^{22} \mathrm{~N}^{2} \mathrm{O}^{2}, \mathrm{HCl} \cdot \mathrm{CHCl}^{3}$ berechnet sich Gesamtchlor: $28,99 \%$, Chlor als $\mathrm{HCl}: 7,24 \%$.

Obgleich der ermittelte Gehalt an Gesamtchlor gegen die Theorie etwas zu niedrig ist, so kann bei der leichten Zersetzbarkeit der vorliegenden Verbindung doch wohl angenommen werden, dass dieselbe im frisch bereiteten Zustande obiger Formel entspricht.

Proben dieser Chloroformverbindung, welche $3-4$ Tage im Exsiccator auf bewahrt waren, ergaben nur noch einen Gesamt-Chlorgehalt von $18,78-23,86 \%$.

Obschon diese Verbindung beim Aufbewahren schon einen Teil des Chloroforms bald wieder abspaltet, ist doch ein anderer Teil desselben ziemlich fest gebunden. So verior z. B. eine Probe obiger Verbindung bei 3 wöchentlichem Liegen an der Luft 14,64\% an Gewicht. Als dieselbe dann 4 Stunden lang auf $100^{\circ} \mathrm{C}$. erhitzt wurde, trat noch ein Gewichtsverlust von $7 \%$ ein, ohne dass damit alles Chloroform schon abgespalten worden wäre. Eine Gewichtskonstanz war erst nach 24 stündigem Erhitzen auf $100^{\circ}$ C. zu erzielen. Einige Proben dieser Verbindung ergaben, nachdem sie 24 Stunden aut $100^{\circ}$ C. erhitzt waren, wobei schliesslich keine Gewichtsabnahme mehr stattfand, bei der Analyse folgende Daten:

1. 0,2062 g Substanz lieferten nach Carius $0,0830 \mathrm{~g} \mathrm{Ag} \mathrm{Cl}=9,95 \% \mathrm{Cl}$.

2. $0,1988 \mathrm{~g}$ Substanz lieferten nach Cariu s $0,0796 \mathrm{~g} \mathrm{Ag} \mathrm{Cl}=9,90 \% \mathrm{Cl}$.

3. 0,3833 g Substanz lieferten nach dem Auflösen in Wasser und Fällen mit Silbernitrat $0,1419 \mathrm{~g} \mathrm{AgCl}=9,16 \% \mathrm{Cl}$.

Berechnet für $\mathrm{C}^{21} \mathrm{H}^{22} \mathrm{~N}^{2} \mathrm{O}^{2}, \mathrm{HCl}$ sind $9,54 \% \mathrm{Cl}$.

Aus obigen Daten geht hervor, dass die Hauptmenge des Chloroforms aus der Verbindung $\mathrm{C}^{21} \mathrm{H}^{22} \mathrm{~N}^{2} \mathrm{O}^{2}, \mathrm{HCl} \cdot \mathrm{CHCI}^{3}$ schon bei gewöhnlicher Temperatur abgegeben wird, dass dagegen die letzten Spuren davon selbst nach längerem Erhitzen auf $100^{\circ} \mathrm{C}$. noch gebunden bleiben. Es erhellt dies besonders aus obigen Analysen 2 und 3, die beide von einem Material, welches 24 Stunden lang bei $100^{\circ} \mathrm{C}$. getrocknet war, ausgefuhrt wurden. Während Gesamtchlor nach Carius $9,90 \%$ gefunden wurde, ergab sich der Chlorgehalt an $\mathrm{HCl}$ nur zu $9,16 \%{ }^{1}$ ).

1) Das Chloroform ist ohne weiteres nicht als ein indifferentes Lösungsmittel für Alkaloide zu betrachten. Sowohl bei der Darstellung von Alkaloiden der verschiedensten Art, als auch bei deren quantitativer Bestimmung in narkotischen Extrakten and Drogen habe ich seit einer Reihe von Jahren die Beobachtung gemacht, dass die durch Ausschüttelung mit Chloroform aus alkalischen Lösungen erhaltenen Basen, nach dem vollständigen Abdestillieren des Lösungsmittels im Wasserbade, mehr oder minder chlorid- und zum Teil auch chloroformhaltig waren.

E. Schmidt.

Arch. d. Pharm. CCXXXVII. Bds. 8. Heft.

40 